PREPARED FOR THE U.S. DEPARTMENT OF ENERGY, UNDER CONTRACT DE-AC02-76CH03073

PPPL-3694

PPPL-3694

UC-70

Effect of Trapped Energetic lons on MHD Activity in Spherical Tori

by

Ya.I. Kolesnichenko, V.V. Lutsenko, V.S. Marchenko, and R.B. White

May 2002

N/M

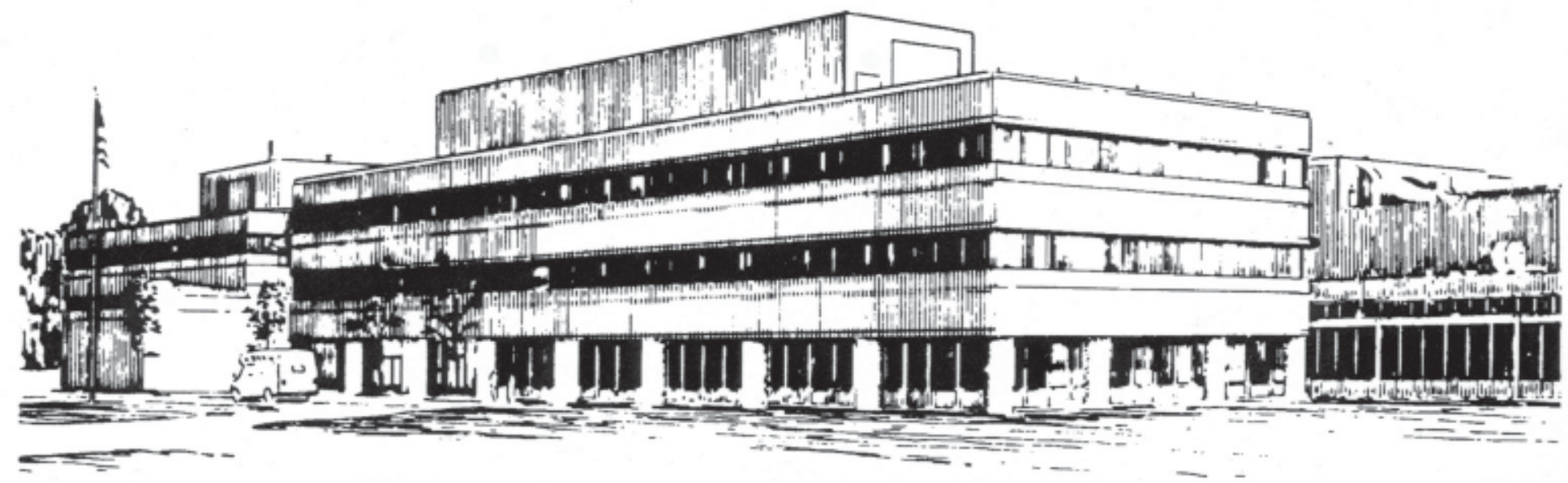

PRINCETON PLASMA PHYSICS LABORATORY PRINCETON UNIVERSITY, PRINCETON, NEW JERSEY 


\section{PPPL Reports Disclaimer}

This report was prepared as an account of work sponsored by an agency of the United States Government. Neither the United States Government nor any agency thereof, nor any of their employees, makes any warranty, express or implied, or assumes any legal liability or responsibility for the accuracy, completeness, or usefulness of any information, apparatus, product, or process disclosed, or represents that its use would not infringe privately owned rights. Reference herein to any specific commercial product, process, or service by trade name, trademark, manufacturer, or otherwise, does not necessarily constitute or imply its endorsement, recommendation, or favoring by the United States Government or any agency thereof. The views and opinions of authors expressed herein do not necessarily state or reflect those of the United States Government or any agency thereof.

\section{Availability}

This report is posted on the U.S. Department of Energy's Princeton Plasma Physics Laboratory Publications and Reports web site in Fiscal Year 2002. The home page for PPPL Reports and Publications is: http://www.pppl.gov/pub_report/

DOE and DOE Contractors can obtain copies of this report from:

U.S. Department of Energy

Office of Scientific and Technical Information

DOE Technical Information Services (DTIS)

P.O. Box 62

Oak Ridge, TN 37831

Telephone: (865) 576-8401

Fax: (865) 576-5728

Email: reports@adonis.osti.gov

This report is available to the general public from:

National Technical Information Service

U.S. Department of Commerce

5285 Port Royal Road

Springfield, VA 22161

Telephone: 1-800-553-6847 or

(703) 605-6000

Fax: (703) 321-8547

Internet: http://www.ntis.gov/ordering.htm 


\title{
Effect of trapped energetic ions on MHD activity in spherical tori
}

\author{
Ya.I. Kolesnichenko ${ }^{1}$, V.V. Lutsenko ${ }^{1}$, V.S. Marchenko ${ }^{1}$, R.B. White ${ }^{2}$ \\ ${ }^{1}$ Institute for Nuclear Research, \\ National Academy of Sciences of Ukraine, Kyiv, 03680, Ukraine \\ ${ }^{2}$ Princeton Plasma Physics Laboratory, P.O. Box 451, Princeton, NJ 08543, USA
}

(May 7, 2002)

\begin{abstract}
It is shown that the increase of $\beta$ (the ratio of plasma pressure to the magnetic field pressure) may change the character of the influence of trapped energetic ions on MHD stability in spherical tori. Namely, the energetic ions, which stabilize MHD modes (such as the ideal kink mode, collisionless tearing mode and semi-collisional tearing mode) at low $\beta$, have a destabilizing influence at high $\beta$ unless the radial distribution of the energetic ions is very peaked.
\end{abstract}

Sawtooth oscillations and internal reconnection events - typical forms of plasma MHD activity - play an important role in Conventional Tokamaks (CT) and Spherical Tori (ST). They redistribute the particles making radial profiles of the plasma parameters flat inside the mixing radius and expelling the energetic ions from the plasma core. The role of such MHD events is especially significant in STs where they can strongly deteriorate the confinement of the energetic ions. ${ }^{1}$ But some particles may be not affected: it was predicted theoretically ${ }^{2}$ and then confirmed experimentally on $\mathrm{TFTR}^{3}$ that trapped ions with the energy above a certain critical magnitude, $\mathcal{E}_{\text {crit }}$, are not sensitive to the sawtooth crash in CTs. The existence of $\mathcal{E}_{\text {crit }}$ is a consequence of the fact that toroidal precession in CTs tends to move particles at $r=$ const, where $r$ is the radial coordinate characterizing the flux surfaces before the crash. On the other hand, such a motion is not compatible with the motion of the bulk plasma across the $r=$ const surfaces during the crash. 
Therefore, the energetic ions stabilize the crash-causing instability when their number is not too small. The situation changes in high- $\beta$ plasmas of STs. As shown in Ref. ${ }^{4}$, plasma diamagnetism results in the "diamagnetic precession", which is directed along the evolving flux surfaces (in contrast to toroidal precession). The diamagnetic precession overrides the toroidal precession when $\beta$ is high $\left(\beta \gtrsim \epsilon\right.$ with $\epsilon=r / R_{o}, R_{o}$ the large radius of the torus), in which case the ions with $\mathcal{E}>\mathcal{E}_{\text {crit }}$ are attached to evolving flux surfaces like the bulk plasma particles. Because of this, one can expect that high energy ions lose their ability to stabilize MHD modes. Furthermore, their effect can even be destabilizing. A more detailed analysis below confirms this conclusion.

Before proceeding to the analysis we note that the stabilizing influence of the energetic ions on MHD modes was observed experimentally on JET, where it was demonstrated that the neutral beam injection may considerably increase the period of sawtooth oscillations. ${ }^{5}$ A relevant theory was suggested in Ref. ${ }^{6}$, where it was concluded that the stabilization is associated with the conservation of the third adiabatic invariant of the particle motion. The latter is conserved when $\omega \ll \omega_{D}$ ( $\omega$ is the mode frequency, $\omega_{D}$ is the precession frequency of the energetic ions), which is essentially equivalent to the condition $\mathcal{E} \gg \mathcal{E}_{\text {crit }}$. This becomes clear if we take into account that $\mathcal{E}_{\text {crit }}$ is determined from the equation $\tau_{\text {crash }}=\omega_{D}^{-1}$ (see Ref. $^{2}$ ) and that $\omega \sim \gamma \gtrsim \tau_{\text {crash }}^{-1}$, where $\gamma$ is the instability growth rate, $\tau_{\text {crash }}$ is the crash duration. However, we emphasize that the mentioned conditions were obtained from different considerations: $\omega \ll \omega_{D}$ is a condition of plasma stability in a linear theory, ${ }^{6}$ whereas $\mathcal{E} \gg \mathcal{E}_{\text {crit }}$ is a condition of unperturbed motion of a test particle during the sawtooth crash, i.e., a condition of the unperturbed motion in an unstable plasma.

We consider first the simplest case of the ideal kink instability described by the following dispersion relation: ${ }^{7}$

$$
-i \frac{\omega}{\omega_{A}}+\widetilde{\mathcal{W}}_{c}+\widetilde{\mathcal{W}}_{h}(\omega)=0
$$

where the first term is the normalized MHD kinetic energy, $\widetilde{\mathcal{W}}_{c}$ is the normalized MHD potential energy of the bulk plasma, $\widetilde{\mathcal{W}}_{h}$ represents the response of the energetic ions, $\omega_{A}=\hat{s} v_{A} /\left.\left(\sqrt{3} R_{0}\right)\right|_{r=r_{s}}, r_{s}$ is the radius of the $q=1$ surface, $\hat{s}$ is the magnetic shear, $v_{A}$ 
is the Alfvén velocity. In particular, in a tokamak with circular flux surfaces the negative of $\widetilde{\mathcal{W}}_{c}$ is ${ }^{8}$

$$
\lambda_{c} \equiv-\widetilde{\mathcal{W}}_{c}=\left(\lambda_{0} / \hat{s}\right) \epsilon_{s}^{2}\left[\beta_{p}^{2}\left(r_{s}\right)-\beta_{c r}^{2}\right]
$$

where $\epsilon_{s}=r_{s} / R_{0}, \lambda_{0}$ the numerical factor of the order of unity, which depends on the $q$ profile, $\beta_{p}\left(r_{s}\right)=\left[8 \pi / B_{p}^{2}\left(r_{s}\right)\right]\left[\bar{p}\left(r_{s}\right)-p\left(r_{s}\right)\right], \bar{p}\left(r_{s}\right)=r_{s}^{-2} \int_{0}^{r_{s}^{2}} p(r), B_{p}$ is the poloidal magnetic field.

In the absence of the energetic ions, Eq. (1) describes the ideal kink instability with the growth rate $\gamma / \omega_{A}=\lambda_{c}$ when $\lambda_{c}>0$. The energetic ions affect this instability. Because the instability is aperiodic, we can assume that the resonant interaction between the energetic ions and the perturbations is absent and, thus, $\widetilde{\mathcal{W}}_{h i}=0$, where the subscript $i$ denotes the imaginary part of a quantity, and take $\widetilde{\mathcal{W}}_{h}(\omega) \equiv-\lambda_{h}(\omega) \approx-\lambda_{h r}(0)$, where the subscript $r$ denotes the real part of the quantity. Therefore,

$$
\frac{\gamma}{\omega_{A}} \approx \lambda_{c}+\lambda_{h r}(0)
$$

It follows from Eq. (3) that, depending on the sign of $\lambda_{h r}(0)$, the effect of the energetic ions is either stabilizing or destabilizing. It was shown in Ref. ${ }^{6}$ that when the population of the energetic ions consists of the trapped particles, $\lambda_{h r}(0)$ is negative for $B \propto 1 / R$ with $R$ the distance from the major axis of the torus, which means that the energetic ions have a stabilizing influence on the ideal kink instability in CTs. However, in high- $\beta$ plasmas of STs $B(R)$ has a minimum ("magnetic valley"), therefore the sign of the response of the energetic ions may change. To see it we write the disturbed part of the distribution function of the fast ions, $\widetilde{f}_{h}$, as follows:

$$
\tilde{f}_{h} \sim \frac{\left(\omega-\omega_{* h}\right) \partial f_{0} / \partial v}{\omega+n \omega_{D}}
$$

where $f_{0}$ is the equilibrium distribution function of the energetic ions, $\omega_{* h} \propto$ $\partial f_{0} / \partial r\left(\partial f_{0} / \partial v\right)^{-1}$ is the diamagnetic frequency of the energetic ions, $n$ is the toroidal mode number. Because $\omega \ll \omega_{D}$, the sign of $\tilde{f}_{h}$ depends on the sign of $\omega_{D}$. The latter is positive for the well trapped particles located in the region with $\partial B / \partial R<0$ (stabilizing particles) and it is negative in the region with $\partial B / \partial R>0$ (destabilizing particles). 
This means that when the particles located at $\partial B / \partial R>0$ mainly contribute to $\lambda_{h r}$, the energetic ions destabilize the ideal kink. Note that finite $\beta$ may have a destabilizing influence on MHD modes even for $\partial B / \partial R<0$, i.e., in CTs, by decreasing the magnitude of the particle trapping parameter, $\kappa_{*}$, separating the regions of particles with $\omega_{D}>0$ and $\omega_{D}<0$ (when $\beta=0, \kappa_{*}$ lies in the region of marginally trapped particles), which enlarges the region of particles with the reversed direction of the precession. ${ }^{9}$

In order to investigate the influence of high $\beta$ on $\lambda_{h r}$ we approximate the magnetic field strength as follows: ${ }^{10}$

$$
B=B_{0}\left[1-\epsilon \cos \theta+\epsilon^{2}\left(\alpha+\sigma \cos ^{2} \theta\right)\right]
$$

where $\alpha$ and $\sigma$ are parameters. In low- $\beta$ plasmas relevant to CTs these parameters are small, but $\alpha \gg 1$ and $\sigma \gtrsim 1$ in STs with high $\beta$. It follows from Eq. (5) that the magnetic valley arises when $\alpha+\sigma>0.5 A$, where $A$ is the aspect ratio of the torus.

For $\lambda_{h r}(0)$ we use the following expression (c.f. Ref. $\left.{ }^{6}\right)$ :

$$
\begin{array}{r}
\lambda_{h r}=-\frac{4 \pi^{3} \epsilon_{s}}{B_{p}^{2}\left(r_{s}\right) \hat{s} r_{s}}\left(\frac{2}{m_{h}}\right)^{3 / 2} \int_{0}^{r_{s}} d r r \int_{\left[1+\epsilon+\epsilon^{2}(\alpha+\sigma)\right]^{-1}}^{\left[1-\epsilon+\epsilon^{2}(\alpha+\sigma)\right]^{-1}} d \lambda \\
\times \lambda I_{b}\langle\cos \theta\rangle\left[1-\frac{\langle\cos \theta\rangle}{\langle\cos \theta\rangle-2 \epsilon\left(\alpha+\sigma\left\langle\cos ^{2} \theta\right\rangle\right)}\right] \int_{0}^{\infty} d \mathcal{E} \mathcal{E}^{3 / 2} \frac{\partial f_{0}}{\partial r}
\end{array}
$$

where $\langle\ldots\rangle$ means bounce averaging, $I_{b} \equiv v \tau_{b} / q R=4 K_{1}(\kappa) /(\epsilon \sqrt{\lambda \sigma}), \kappa$ is the particle trapping parameter, $\lambda=\mu B_{0} / \mathcal{E}, m_{h}$ is the ion mass, $K_{1}(\kappa)$ and $\kappa$ are defined in Ref. ${ }^{10}$ The results of the calculation of $\Lambda_{h r} \equiv \lambda_{h r}(0) \hat{s} /\left(\epsilon_{s} \beta_{p h}\right)$, where $\beta_{p h}=8 \pi p_{h} / B_{p}^{2}$ with $p_{h}$ is the fast ion pressure, for an NSTX-like torus with $r_{s} / R_{0}=1 / 3$ are shown in Fig. 1 . We conclude from here that $\lambda_{h r}(0)>0$ for $\alpha>\alpha_{\text {crit }}\left(r_{s} / r_{h}\right)$, where $r_{h}$ is a characteristic width of the radial profile of the energetic ions, $\alpha_{\text {crit }}$ growing with $r_{s} / r_{h}$. The obtained dependence of $\alpha_{c r i t}$ on $r_{s} / r_{h}$ is explained by the facts that there is a minimum of $B(R)$ at $r \leq r_{s}$ only in the case when $\alpha \geq 0.5$ and that the region with $\partial B / \partial R>0$ is sufficiently wide only for $\alpha$ well exceeding 0.5 , see Fig. 2. When $\alpha \gtrsim 0.5$, the region with $\partial B / \partial R>0$ near $r_{s}$ is narrow. Therefore, the effect of fast ions can be destabilizing provided that $n_{h}(r)$ is flat. In the case of $\alpha \gg 0.5$ the trapped particles with $\omega_{D}<0$ dominate in the region of the localization of the ideal kink mode even when the radial profile of the energetic ions is strongly peaked. In addition, it follows from Fig. 1 that the destabilizing 
effect of the energetic ions is largest when $r_{h} \sim r_{s}$. This is explained by the fact that the fast particle drive is proportional to $d n_{h} / d r$ (which leads to small $\lambda_{h r}$ at $r_{h} \gg r_{s}$ ) and, on the other hand, it is proportional to the number of fast particles with the negative precession frequency (which leads to small $\lambda_{h r}$ at $r_{h} \ll r_{s}$ ).

Equation (1) ignores the finite magnitude of the bulk ion diamagnetic frequency, $\omega_{*}$. When finite $\omega_{*}$ is taken into account and, in addition, $\lambda_{c}+\lambda_{h r}>0$, the dispersion relation has the form: ${ }^{11}$

$$
\left[\omega\left(\omega-\omega_{*}\right)\right]^{1 / 2}=i \omega_{A}\left[\lambda_{c}+\lambda_{h r}(\omega)\right]
$$

Assuming that $\omega \ll \omega_{D}$, we can take again $\lambda_{h r}(\omega)=\lambda_{h r}(0)$. Then Eq. (7) yields:

$$
\omega=\frac{\omega_{*}}{2} \pm i \omega_{A}\left\{\left[\lambda_{c}+\lambda_{h r}(0)\right]^{2}-\left(\frac{\omega_{*}}{2 \omega_{A}}\right)^{2}\right\}^{1 / 2} .
$$

We conclude from Eq. (8) that a plasma without the energetic ions is unstable when $\lambda_{c}^{2}>\left(0.5 \omega_{*} / \omega_{A}\right)^{2}$. The energetic particles have a stabilizing influence on the instability when $\lambda_{h r}(0)<0$, the instability being completely stabilized when

$$
\omega_{A}\left|\lambda_{c}+\lambda_{h r}(0)\right|<0.5\left|\omega_{*}\right|
$$

However, when $\lambda_{h r}(0)>0$, the energetic ions play a destabilizing role.

In STs the ion Larmor radius $\left(\rho_{i}\right)$ is rather large. If it exceeds the width of the ideal inertial layer, $\rho_{i} / r_{s}>\lambda_{c}$, non-ideal modes accompanied by the reconnection of the magnetic field lines in the vicinity of the $q=1$ radius can be destabilized (we consider a plasma with a monotonic $q(r))$. Typically, in STs the ion Larmor radius exceeds both the width of the resistive reconnection layer, $\delta_{\text {res }}\left[\delta_{\text {res }} \sim r_{s} S_{M}^{-1 / 3} \hat{s}^{-1 / 3}\right.$, where $S_{M}$ is the magnetic Reynolds (Lundquist) number] and the electron skin depth, $d_{e}\left(d_{e} \equiv c / \omega_{p e}, \omega_{p e}\right.$ is the electron plasma frequency). This implies that the collisionless reconnection driven by either the collisionless tearing mode or semi-collisional tearing mode may occur in STs. Note that presumably these instabilities are responsible for the sawtooth crashes in tokamaks. The corresponding dispersion relation can be written as follows: ${ }^{12}$

$$
\frac{\pi}{2}\left(\frac{\gamma}{\omega_{A}}\right)^{2}=\frac{\rho_{\tau}}{r_{s}}\left[\lambda_{c}+\lambda_{h r}(0)\right]+\frac{\rho_{\tau}^{2} d_{e}}{r_{s}^{3}} \frac{\omega_{A}}{\gamma} \sqrt{1+\frac{\nu_{e i}}{2 \gamma}}
$$


$\rho_{\tau}=(1+\tau)^{1 / 2} \rho_{i}$ with $\tau=\left(T_{e} / T_{i}\right)_{r_{s}}$.

In the limit case of $\nu_{e i} \ll \gamma$ Eq. (10) describes the collisionless tearing instability. It can be stabilized by finite $\omega_{*}$ when ${ }^{12}$

$$
\left(\frac{m_{i}}{m_{e}}\right)^{1 / 6}\left(\frac{\beta_{e}}{2}\right)^{2 / 3} \frac{L_{s}}{L_{n}}>1,
$$

where $L_{s}=q R / \hat{s}, \hat{s}$ the magnetic shear, and $L_{n} \equiv\left|d \ln n_{e} / d \ln r\right|^{-1}$. We, however, assume that Eq. (11) is not satisfied.

When a plasma is on the margin of the ideal MHD stability $\left(\lambda_{c} \approx 0\right)$ and $\lambda_{h}=0$, the growth rate of the collisionless tearing instability is ${ }^{12}$

$$
\frac{\gamma_{0}}{\omega_{A}}=\left(\frac{2}{\pi}\right)^{1 / 3} \frac{\left(d_{e} \rho_{\tau}^{2}\right)^{1 / 3}}{r_{s}},
$$

which is valid for $\left|\lambda_{c}\right| \ll\left(\rho_{i} d_{e}^{2}\right)^{1 / 3} / r_{s}$. In the presence of a small number of the fast ions we find:

$$
\gamma=\gamma_{0}\left[1+\left(\frac{2}{\pi}\right)^{1 / 3} \frac{\left(\lambda_{c}+\lambda_{h r}(0)\right) r_{s}}{3\left(d_{e}^{2} \rho_{\tau}\right)^{1 / 3}}\right] .
$$

We observe that the effect of the energetic ions with $\lambda_{h r}(0)>0$ is destabilizing. But when the number of the energetic ions is sufficiently large, the condition of applicability of Eq. (13) is broken and, thus, an additional analysis is required.

Below we consider the limit case of

$$
\left|\lambda_{c}+\lambda_{h r}(0)\right| \gg\left(\rho_{\tau} d_{e}^{2}\right)^{1 / 3} / r_{s}
$$

If we neglect the LHS term in Eq. (10), we can see that a plasma below the ideal MHD stability limit $\left(\lambda_{c}<0\right)$ is unstable in the absence of the energetic ions $\left(\lambda_{h r}=0\right)$. Keeping the term with $\lambda_{h r}$, we obtain the following growth rate of the collisionless tearing mode:

$$
\frac{\gamma}{\omega_{A}}=-\frac{\rho_{\tau} d_{e}}{r_{s}^{2}} \frac{1}{\lambda_{c}+\lambda_{h r}(0)}
$$

We see that the energetic ions with $\lambda_{h r}(0)<0$ tend to stabilize the instability in a plasma with $\lambda_{c}<0$. On the contrary, when $\lambda_{h r}(0)>0$, the instability is enhanced. Formally, $\gamma \rightarrow \infty$ for $\lambda_{c}+\lambda_{h r}(0)=0$, but then Eq. (14) is broken. When $\lambda_{c}+\lambda_{h r}(0)>0$, Eq. (15) yields $\gamma<0$, which, however, does not mean that a plasma is stable because Eq. (15) was obtained neglecting the term proportional to $\gamma^{2}$. 
On the other hand, Eq. (15) predicts that the energetic ions can drive the instability in a low- $\beta$ plasma when $\lambda_{h r}(0)<0$ and $\lambda_{c}>0$. But this instability is weak. Indeed, the first term in the LHS of Eq. (10) is negligible when $\gamma / \omega_{A} \ll\left(\rho_{\tau}^{2} d_{e}\right)^{1 / 3} / r_{s}$, which together with Eq. (15) yields $\gamma / \omega_{A} \ll \sqrt{\rho_{\tau}\left|\lambda_{c}+\lambda_{h r}(0)\right| / r_{s}}$. Probably, this instability is stabilized by some factors, which are not taken into account in Eq. (10).

In the most interesting case of strong instability the term in Eq. (10) proportional to $\gamma^{2}$ cannot be neglected. Then one can see that the effect of $\lambda_{h r}(0)>0$ is destabilizing. In particular, when the last term in Eq. (10) is negligible, we find:

$$
\frac{\gamma}{\omega_{A}}=\sqrt{\frac{2}{\pi} \frac{\rho_{\tau}}{r_{s}}\left[\lambda_{c}+\lambda_{h r}(0)\right]}
$$

Equation (16) shows that when $\beta$ is high, the energetic ions enhance the instability in a plasma with $\lambda_{c}>0$ or even can lead to the instability in a plasma with $\lambda_{c}<0$. We recall that the considered collisionless tearing instability occurs when

$$
\gamma / \omega_{A}<\rho_{\tau} / r_{s}, \quad \delta_{\text {res }}<d_{e}<\rho_{\tau}
$$

The semi-collisional tearing instability may occur when $\rho_{\tau}>\delta_{\text {res }}>d_{e}$, which implies that $\nu_{e i}>\gamma$. In this case when $\lambda_{c} \approx 0$ and $n_{h}=0$, Eq. (10) yields ${ }^{13}$

$$
\frac{\gamma_{0}}{\omega_{A}}=\left(\frac{2}{\pi}\right)^{2 / 7}\left(\frac{\rho_{\tau}}{r_{s}}\right)^{4 / 7} S_{M}^{-1 / 7} \hat{s}^{-1 / 7}
$$

In the presence of the energetic ions when

$$
\lambda_{c}+\lambda_{h r}(0) \gg\left(\rho_{i} / r_{s}\right)^{1 / 7} S_{M}^{-2 / 7} \hat{s}^{-2 / 7}
$$

and the last term in Eq. (10) is negligible, the growth rate is given again by Eq. (16). This means that the collisionless tearing instability and semi-collisional tearing instability are characterized by the same growth rate when

$$
\lambda_{c}+\lambda_{h r}(0) \gg \max \left\{\left(\rho_{\tau} d_{e}^{2}\right)^{1 / 3} / r_{s},\left(\rho_{i} / r_{s}\right)^{1 / 7} S_{M}^{-2 / 7} \hat{s}^{-2 / 7}\right\}
$$

Equation (10) predicts also a weak semi-collisional tearing instability driven by the energetic ions at low $\beta$ when $\lambda_{c}+\lambda_{h r}(0)<0, \lambda_{c}>0$ :

$$
\frac{\gamma}{\omega_{A}}=\left(-\frac{1}{\lambda_{c}+\lambda_{h r}(0)}\right)^{2 / 3}\left(\frac{\rho_{\tau}}{r_{s}}\right)^{2 / 3} \hat{s}^{-1 / 3} S^{-1 / 3} \text {. }
$$


It is of interest to make numerical estimates for NSTX. We take $B=0.3 T, a=68 \mathrm{~cm}$, $A=1.3, n=3 \times 10^{13} \mathrm{~cm}^{-3}, T=1 \mathrm{keV}, k=2$ ( $k$ is the elongation of the flux surfaces), $r_{s}=a / 2, \hat{s}=0.5, \mathcal{E}_{b}=80 \mathrm{keV}$. Then $\rho_{i}=2.15 \mathrm{~cm}, \delta_{\text {res }}=0.33 \mathrm{~cm}$, and $d_{e}=0.1 \mathrm{~cm}$, which implies that semi-collisional tearing instability may occur. However, the ratio $\delta_{\text {res }} / d_{e}$ depends on plasma parameters in a way that the regime of the collisionless tearing mode can take place in a plasma with the same $\beta$ as in the considered above example. Indeed, $\delta_{\text {res }} / d_{e} \propto n^{2 / 3} T^{-1 / 2} B^{-1} \hat{s}^{-1 / 3}\left(R_{0} r_{s}\right)^{1 / 3}$, therefore, increasing the temperature by, e.g., a factor of 4 and keeping $n T=$ const, we obtain the regime with $\delta_{r e s}<d_{e}$.

In conclusion, we have shown for the first time that the increase of $\beta$ may deprive the trapped energetic ions of their ability to stabilize MHD modes in STs. This is shown for the ideal kink mode and the non-ideal modes, in particular, for the collisionless tearing mode and semi-collisional tearing mode. Moreover, we have found that the energetic ions may have the destabilizing influence on the mentioned modes. Because of this, the trapped energetic ions must trigger the sawtooth crashes in high- $\beta$ plasmas of STs rather than to prevent them as is the case in CTs. The destabilization occurs for $\beta \gtrsim \epsilon_{s}$, i.e., when the equilibrium magnetic field is characterized by the presence of the magnetic valley. The destabilizing influence of the energetic ions is strongest when the radial profile of the energetic ions is flat enough to provide the dominant contribution of particles with the negative precession frequency to the response $\lambda_{h r}(0)$ but, on the other hand, it must be sufficiently peaked to maximize the instability drive by $\nabla n_{h}$. The obtained results together with the prediction of the stabilization of the fishbone mode in STs with high $\beta^{14,10,15}$ indicate that the physical picture of MHD activity in plasmas with fast ions may strongly vary with $\beta$.

\section{ACKNOWLEDGEMENTS}

The research described in this Letter was made possible in part by the International Atomic Energy Agency Research Contract No. 10539, Award No. UP2-2419-KV-02 of the US Civilian Research and Development Foundation, and DoE contract DE-AC02-76CH03073. The authors thank C.Z. Cheng for a discussion of the results of the work. 


\section{REFERENCES}

${ }^{1}$ S.S. Medley, A.L. Roquemore, "Neutral Particle Analyzer Measurements of Ion Behavior in NSTX", NSTX Results Rewiew, PPPL, September, 2001.

${ }^{2}$ Ya.I. Kolesnichenko, Yu.V. Yakovenko, Report at the 4th IAEA TC Meeting on Alpha Particles in Fusion Research, (Princeton, April 1995); Nuclear Fusion 36, 159 (1996).

${ }^{3}$ N.N. Gorelenkov, R.V. Budny, H.H. Duong, et al., Nuclear Fusion 37, 1053 (1997).

${ }^{4}$ Ya.I. Kolesnichenko, V.V. Lutsenko, R.B. White, Yu.V. Yakovenko, Phys. Letters A 287, 131 (2001).

${ }^{5}$ D.J. Campbell, et al., Phys. Rev. Lett. 60, 2148 (1988).

${ }^{6}$ F. Porcelli, Plasma Phys. Contr. Fusion 33, 1601 (1991).

${ }^{7}$ R.B. White, "The theory of toroidally confined plasmas", Imperial Colledge Press, 2001.

${ }^{8}$ M.N. Bussac, R. Pellat, D. Edery, J.L. Soule, Phys. Rev. Lett. 35, 1638 (1975).

${ }^{9}$ Yanlin Wu, C.Z. Cheng, and R.B. White, Phys. Plasmas 1, 3369 (1994).

${ }^{10}$ Ya.I. Kolesnichenko, V.V. Lutsenko, V.S. Marchenko, Nucl. Fusion 40, 1731 (2000).

${ }^{11}$ B. Coppi, P. Detragiache, S. Migliuolo, F. Pegoraro, F. Porcelli, Phys. Rev. Lett. 63, $2733(1989)$.

${ }^{12}$ F. Porcelli, Phys. Rev. Letters 66, 425 (1991).

${ }^{13}$ F. Porcelli, D. Boucher and M.N. Rosenbluth Plasma Phys. Contr. Fusion 38, 2163 (1996).

${ }^{14}$ Ya.I. Kolesnichenko, V.V. Lutsenko, V.S. Marchenko, Phys. Rev. Lett. 82, 3260 (1999).

${ }^{15}$ Ya.I. Kolesnichenko, V.S. Marchenko, R.B. White, Phys. Plasmas 8, 3143 (2001). 
FIGURES

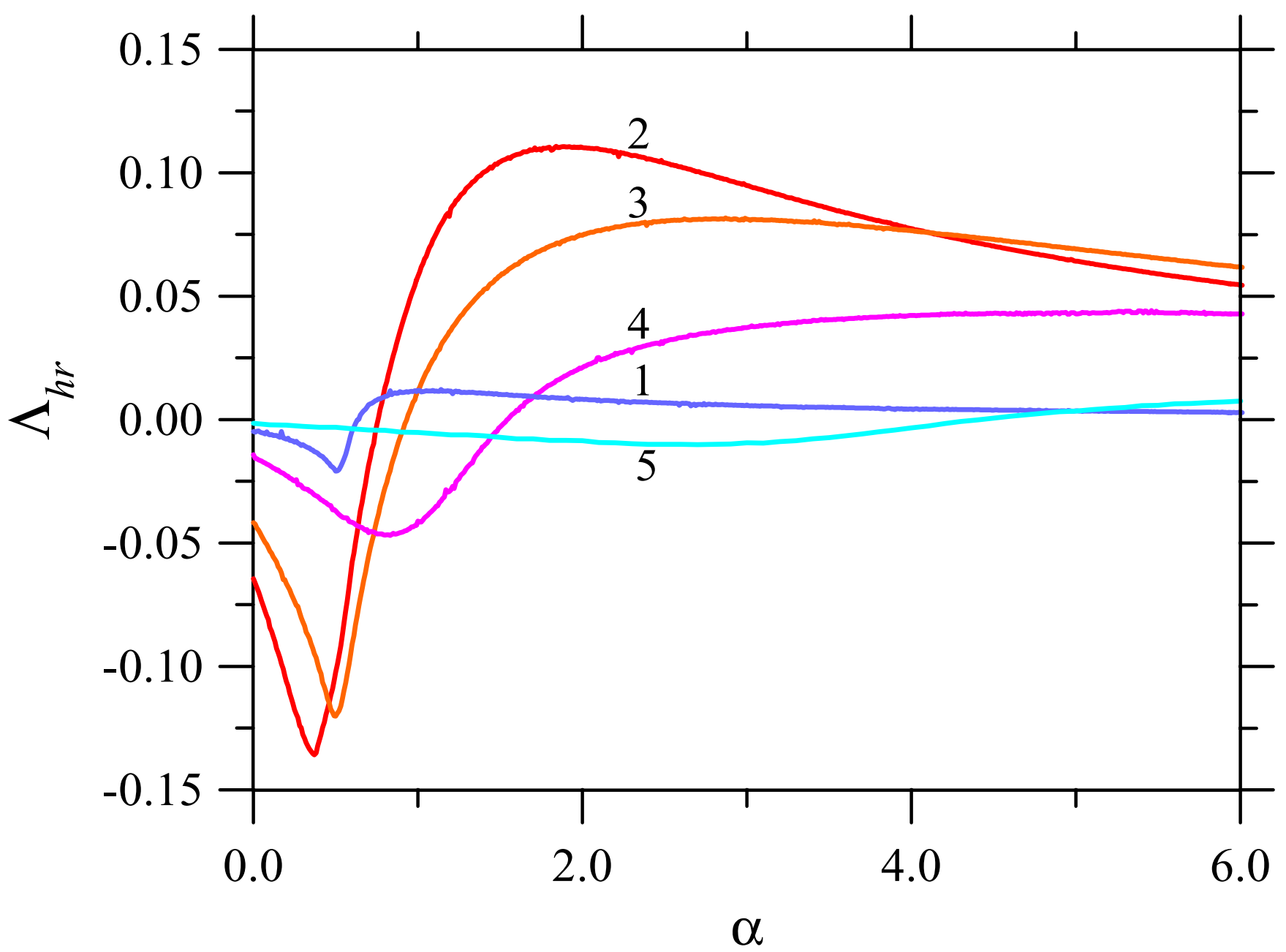

FIG. 1. $\Lambda_{h r} \equiv \lambda_{h r}(0) \hat{s} /\left(\epsilon_{s} \beta_{p h}\right)$ versus $\alpha$ in a spherical torus with $A=1.3, \epsilon_{s}=1 / 3, \sigma=1$ for various $r_{s} / r_{h}: 1-0.2,2-1.2,3-2,4-3,5-7$. Here $r_{h}$ characterizes the radial profile of the energetic ions taken in the form: $n_{h}(r)=n_{h}(0) \exp \left(-r^{2} / r_{h}^{2}\right)$. The region of $\alpha \geq 0.5$ corresponds to an equilibrium configuration with the minimum of the magnetic field at $r \leq r_{s}$. 


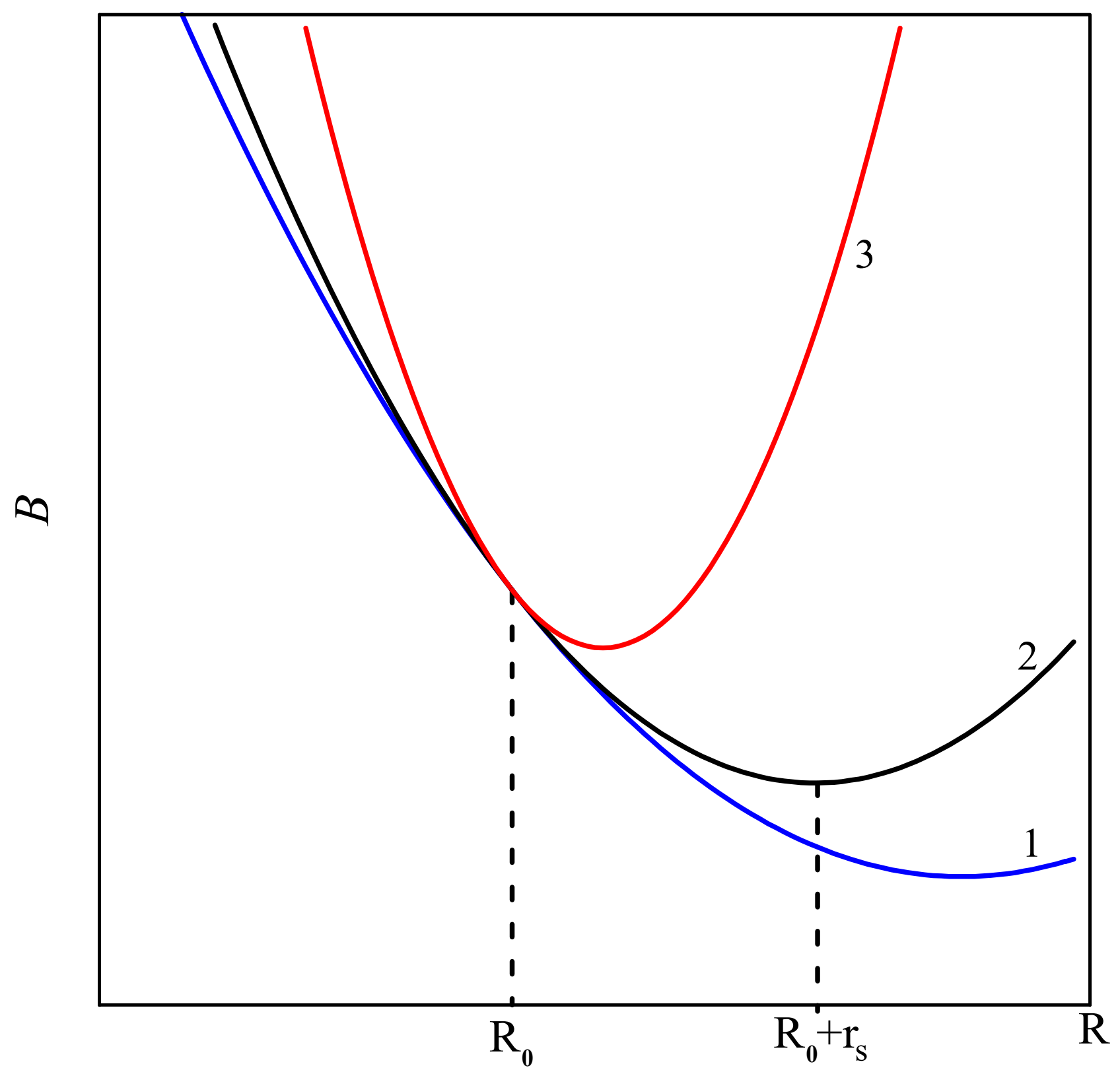

FIG. 2. The equilibrium magnetic field strength with a magnetic valley for various $\alpha: 1-\alpha=0.01,2-\alpha=0.5,3-\alpha=4$. The magnetic field is described by $B=B_{0}\left[1-\epsilon \cos \theta+\epsilon^{2}\left(\alpha+\cos ^{2} \theta\right)\right]$, and $\epsilon_{s}=1 / 3$. 


\section{External Distribution}

Plasma Research Laboratory, Australian National University, Australia

Professor I.R. J ones, Flinders University, Australia

Professor J oão Canalle, Instituto de Fisica DEQ/IF - UERJ , Brazil

Mr. Gerson O. Ludwig, Instituto Nacional de Pesquisas, Brazil

Dr. P.H. Sakanaka, Instituto Fisica, Brazil

The Librarian, Culham Laboratory, England

Library, R61, Rutherford Appleton Laboratory, England

Mrs. S.A. Hutchinson, JET Library, England

Professor M.N. Bussac, Ecole Polytechnique, France

Librarian, Max-Planck-Institut für Plasmaphysik, Germany

J olan Moldvai, Reports Library, MTA KFKI-ATKI, Hungary

Dr. P. Kaw, Institute for Plasma Research, India

Ms. P.J . Pathak, Librarian, Insitute for Plasma Research, India

Ms. Clelia De Palo, Associazione EURATOM-ENEA, I taly

Dr. G. Grosso, Instituto di Fisica del Plasma, Italy

Librarian, Naka Fusion Research Establishment, J AERI, J apan

Library, Plasma Physics Laboratory, Kyoto University, J apan

Research Information Center, National Institute for Fusion Science, J apan

Dr. O. Mitarai, Kyushu Tokai University, J apan

Library, Academia Sinica, Institute of Plasma Physics, People's Republic of China

Shih-Tung Tsai, Institute of Physics, Chinese Academy of Sciences, People's Republic of China

Dr. S. Mirnov, TRINITI, Troitsk, Russian Federation, Russia

Dr. V.S. Strelkov, Kurchatov Institute, Russian Federation, Russia

Professor Peter Lukac, Katedra Fyziky Plazmy MFF UK, Mlynska dolina F-2, Komenskeho Univerzita, SK-842 15 Bratislava, Slovakia

Dr. G.S. Lee, Korea Basic Science Institute, South Korea

Mr. Dennis Bruggink, Fusion Library, University of Wisconsin, USA

Institute for Plasma Research, University of Maryland, USA

Librarian, Fusion Energy Division, Oak Ridge National Laboratory, USA

Librarian, Institute of Fusion Studies, University of Texas, USA

Librarian, Magnetic Fusion Program, Lawrence Livermore National Laboratory, USA

Library, General Atomics, USA

Plasma Physics Group, Fusion Energy Research Program, University of California at San Diego, USA

Plasma Physics Library, Columbia University, USA

Alkesh Punjabi, Center for Fusion Research and Training, Hampton University, USA

Dr. W.M. Stacey, Fusion Research Center, Georgia Institute of Technology, USA

Dr. J ohn Willis, U.S. Department of Energy, Office of Fusion Energy Sciences, USA

Mr. Paul H. Wright, Indianapolis, Indiana, USA 
The Princeton Plasma Physics Laboratory is operated by Princeton University under contract with the U.S. Department of Energy.

\author{
Information Services \\ Princeton Plasma Physics Laboratory \\ P.O. Box 451 \\ Princeton, NJ 08543
}

Phone: 609-243-2750

Fax: 609-243-2751

e-mail: pppl_info@pppl.gov

Internet Address: http://www.pppl.gov 